\title{
An Attempt at MOOC Localization for Chinese-Speaking Users
}

\author{
Xiaoyin Che, Sheng Luo, Cheng Wang, and Christoph Meinel
}

\begin{abstract}
Internetworking with TCP/IP" is a massive open online course (MOOC) provided by Germany-based MOOC platform "openHPI", which has been offered in German, English and - recently - Chinese respectively, with similar content. In this paper, the authors, who worked jointly as a teacher (or as teaching assistants) in this course, want to share their ideas derived from daily teaching experiences, analysis of the statistics, comparison between the performance in different language offers and the feedback from user questionnaires. Additionally, the motivation, attempt and suggestion at MOOC localization will also be discussed.
\end{abstract}

Index Terms - Chinese-speaking users, localization, MOOC, openHPI.

\section{INTRODUCTION}

The massive online open course (MOOC) is an explosively developing form of e-learning that has taken place in recent years. The initial model of MOOC was first proposed in 2008 [1]. Only a few years later, particularly in 2012, with "the year of the MOOC" [2], it has expanded worldwide at an incredible speed. By now, many top universities and educational organizations are involved in the wave of MOOCs, which offers great opportunities to the learners from all corners of the world.

OpenHPI is a Germany-based non-profit MOOC platform, concentrating on information technology and hosted by Hasso Plattner Institute (HPI), in Potsdam, Germany [3]. According to the categories of cMOOC and xMOOC, openHPI is a typical xMOOC platform. The course language can be English or German. And the basic unit of the course is a week, which consists of lecture videos, ungraded self tests and a graded homework. All the course participants need to follow the same schedule. The final grade of a participant is based on the sum of all his/her scores in weekly homework and a final exam. The certificate of participation and the record of achievement are granted to the learners who complete the course.

The first openHPI course started in September 2012, and by February 2014, the total course enrollments reached 100000. And also in February 2014, the first openHPI course with Chinese as the teaching language was offered on openHPI.cn, a sub-platform of openHPI hosted in Shanghai, China. The idea of setting up openHPI.cn was introduced at about the same time as the establishment of the main platform openHPI.de, because the director of the openHPI program noticed that the number of potential MOOC users in China is huge. However, considerable obstacles prevent the learners

Manuscript received August 9, 2014; revised October 10, 2014.

The authors are with the Hasso Plattner Institute, Potsdam, Germany (e-mail: xiaoyin.che@hpi.de). who live in mainland China to join the course on openHPI.de.

In order to offer better service on openHPI.cn, we don't want to simply clone the courses from openHPI.de. Localizing them will not only help the Chinese learners understand the content, but also show our sincerity. And through the experience gained as the members of openHPI.cn teaching team, we have learned that a localized course should be more than a simply translated course. Just providing subtitles is far from enough.

The authors worked jointly as a teacher or as teaching assistants in the course "Internetworking with TCP/IP", simplified as "Internetworking", which was available on both openHPI.de and openHPI.cn. From 2012 to 2014, this course has been offered three times with similar content, but in German, English and Chinese respectively. The basic information of these "Internetworking" courses can be found in Table I. In this paper, we will try to first analyze the users' behavior and the reasons behind them, and then propose our solution or suggestion. The main source of statistics comes from the forums and the questionnaires.

The rest of this paper is arranged as follow. In Section II we will discuss our motivation and why localization efforts can help. Our attempt will be illustrated in detail in Section III while the conclusion and future work can be found in Section IV.

\section{Motivation}

Not everyone is a perfectionist, but "striving for excellence is a normal, innate aspect of human development" [4]. This is why numerous young students, especially those from developing countries, strive for the chance to receive higher education from the highly ranked universities in developed countries. When they want to learn, they want to learn from the best. Unfortunately, the educational resources in such places are limited, and as such can never meet the huge demand. However, E-learning, MOOCs in particular, theoretically provides the opportunity to everyone.

But on the practical level, things are not that simple. As the MOOC provider, it is a tremendous challenge to offer a course simultaneously to hundreds of thousands of learners with highly diverse fundamental knowledge, learning habits, participating purposes and cultural backgrounds. And on the learner side, the situation is even more complicated involving: curiosity vs. uncertainty, confidence vs. frustration, expectation vs. disappointment, determination vs. slackness, etc. And for the learners from most developing countries [5], [6], for example China, all the difficulties are amplified by an additional but conspicuous factor: language.

At the same time, the development of MOOCs in China is also very fast [7]. By now it's not difficult to find a 
China-based MOOC platform offering courses with Chinese as the language of instruction. To attend a Chinese course is indeed a good option for the Chinese learners, no need to face the language barrier and no fear of confusion in an alien learning culture. But on the other hand, quite a lot of learners are not fully satisfied with this choice. As we mentioned at the beginning of this section, people want the best. Apparently in the world of higher education, especially in the area of science and technology, China does not have the best resources, yet.

TABLE I: THREE "INTERNETWORKING" COURSES

\begin{tabular}{|c|c|c|c|c|c|c|c|c|}
\hline Platform & Start Date & Language & Enrollments & Certificate Earners & Completion Rate & Forum Stats & Questionnaire & Q-Respondents \\
\hline openHPI.de & 04.11 .2012 & German & 9891 & 1635 & $16.5 \%$ & $\times$ & $\sqrt{ }$ & 1584 \\
\hline openHPI.de & 31.03 .2014 & English & 5268 & 836 & $15.9 \%$ & $\sqrt{ }$ & $x$ & - \\
\hline openHPI.cn & 16.06 .2014 & Chinese & 1650 & 84 & $5.1 \%$ & $\sqrt{ }$ & $\sqrt{ }$ & 99 \\
\hline
\end{tabular}

Then here comes ambivalence among the potential Chinese MOOC users. On the one hand, they want to attend the original top-level foreign courses and be treated exactly the same as the participants from the western world. On the other hand, many of them have difficulties or are unsure about their own capabilities to successfully finish the courses. This point of view was supported by the feedback questionnaire results submitted by the user group of the openHPI course "Internetworking". The relevant investigation item and its result can be found in Table II and Fig. 1.

TABLE II: THE QUESTIONNAIRE ITEM ON THE WILLINGNESS OF JOINING INTERNATIONAL PLATFORM AND ITS RESULTS

\begin{tabular}{lcc}
\hline \hline $\begin{array}{l}\text { When possible, do you want to join openHPI.de and } \\
\text { learn with the participants all over the world? }\end{array}$ & Count & Ratio \\
\hline $\begin{array}{l}\text { (I) Yes, absolutely } \\
\text { (II) Yes, but I can only give a try since my foreign }\end{array}$ & 34 & $58.6 \%$ \\
$\begin{array}{l}\text { language is not very good } \\
\text { (III) No, because I know my foreign language ability }\end{array}$ & 6 & $6.1 \%$ \\
$\begin{array}{l}\text { is not adequate for that } \\
\text { (IV) No, the language is not the problem for me, but I } \\
\text { just prefer localized course. }\end{array}$ & 1 & $1.0 \%$ \\
\hline \hline
\end{tabular}

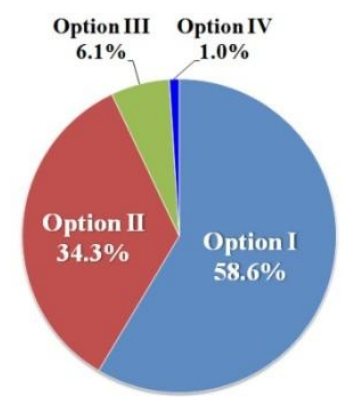

a) Full scale

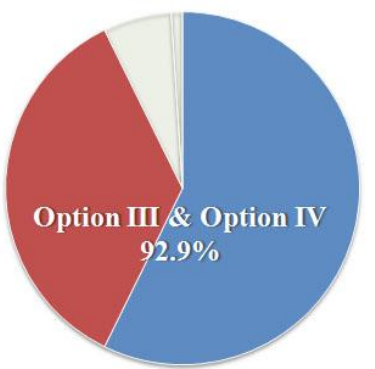

b) Who wants to join

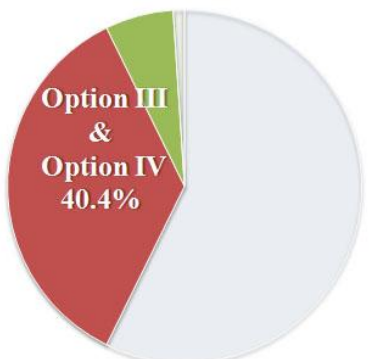

c) Who concerns own capability
Based on the questionnaire, over $90 \%$ of respondents intend to join openHPI.de - the openHPI main platform that offers more courses in English or German with a larger international user group (Fig. 1b). And there are also about
$40 \%$ of the respondents (Fig. 1c) who doubt whether their language skill is good enough to attend the international courses, including $34.3 \%$ who still want to give it a try and another $6.1 \%$ who don't. Only one respondent suggests he/she prefers translated course in Chinese, although having no problem with the original course language.

We attempt to solve this ambivalence by localizing the course. The content should be kept as close to the original as possible, while its manifestation changes. We aim to not only satisfy some Chinese learners' psychological desire to attend original openHPI course from Germany, but also to support their efforts by improvements in the all-around localization. Some previous works in localization [7]-[9] have already been launched. Today even Coursera provides multilingual subtitles for some of its courses to help the non-native English speakers. In this paper, we suggest that while localization starts with translation, it must be more.

\section{ATTEMPT OF OPENHPI.CN}

\section{A. Breaking the Language Barrier}

When talking about localization, language is always on top of the to-do-list. According to the stats reported from edX's English-speaking course " $6.002 \mathrm{x}$ ", the number of Chinese enrollees - 622 altogether and about $0.4 \%$ of total participants - was far below the expectation [10]. On the other hand, over 13000 learners from India registered "6.002x" [11]. China and India, as the two largest developing countries in the world, share the similar desire for better quality higher education. But their difference in participating in " $6.002 \mathrm{x}$ " is huge, why? The reason must be complicated, but we believe language is a key factor.

No one can participate in a course without understanding the course language. English is the co-official language in India, which results in the English penetration rate there being obviously higher than in China, especially when excluding the students who can speak adequate English but study abroad. Therefore it's quite understandable that many more IP addresses have been detected in India that accessed the English-speaking course "6.002x".

It's quite hard to figure out or estimate how many Chinese people can speak English, not to mention how many of them are capable of attending an English-speaking course [12], [13]. The language barrier, mainly English but not always, is what lies between the high quality courses and many knowledge-hungry learners. Breaking the language barrier is the first task in the localization effort. 
Since it's not realistic to expect everyone to suddenly have the capability necessary to master the original course language, the only choice is to provide proper translation due to targeted user group, which is Chinese in our case. It is a well-known fact, that Chinese is completely different from any western language. In order to avoid confusing the user, a proper form of translation need to be found.

Curtin University in Australia offered a bilingually delivered MOOC: Australia China Trade (ACT) in 2014 [14]. In their effort the researchers there provided 4 different potential solutions to solve the bilingual delivery problem:

- English video with Chinese subtitle

- Recording two videos in English and Chinese respectively

- Chinese video with English transcript

- One video with an additional audio track in the other language

These four options cover almost all the needs of both Chinese-speaking and English-speaking course participants. For openHPI.cn, Chinese-speaking users are the only target group. In the "Internetworking" course, our preparation time was limited and as a result we only embedded the Chinese subtitles. However, because we want to know which solution is most welcomed by our users, we have implemented an investigation item in the user feedback questionnaire. The result is shown in Table III and Fig. 2.

TABLE III: THE QUESTIONNAIRE ITEM ON THE PREFERENCE OF LECTURE VIDEO LANGUAGE DELIVERY AND ITS RESULTS

\begin{tabular}{lcc}
\hline \hline $\begin{array}{l}\text { When the lecturer gives the course on openHPI.cn in a } \\
\text { foreign language, which form of language delivery in } \\
\text { the lecture video do you prefer? }\end{array}$ & Count & Ratio \\
\hline $\begin{array}{l}\text { (I) Absolutely in original language, nothing more } \\
\text { (II) In original language, but adding Chinese subtitles }\end{array}$ & 19 & $19.2 \%$ \\
$\begin{array}{l}\text { (III) In original language, but adding bilingual subtitles } \\
\text { (original language and Chinese) }\end{array}$ & 36 & $36.4 \%$ \\
(IV) In original language. If the teaching language is & 28 & $28.3 \%$ \\
$\begin{array}{l}\text { English, please add bilingual subtitles, or else, only } \\
\text { Chinese-subtitles is enough }\end{array}$ & 5 & $5.0 \%$ \\
(V) Dubbing the lecture video into Chinese & $5.1 \%$ \\
\hline \hline
\end{tabular}

From the stats we can easily find out that the majority of the respondents (about 84\%, as shown in Fig. 2b) prefer the combination of original audio track and additional subtitles. And among them bilingual subtitles is the most desired option, selected by around 2/3 of all the respondents (Fig. 2c). There are two main reasons for this phenomenon. The first is that bilingual subtitles allow the learners with basic foreign language skills (which is quite common in the user group) to understand the course better, especially in views of the specialized vocabularies. And the second reason is that the learners could possibly improve their foreign language skill by carefully studying the correctly translated bilingual subtitles. In this way, it would also be a great success for us, as the course providers, to kill two birds with one stone.

Furthermore, there are also some users who prefer to have even more thoroughly localized lecture videos by changing the audio track $(5.1 \%)$ or even the lecturer $(7.1 \%)$. Just as some others prefer a purely original course $(4.0 \%)$. One single offer can never satisfy everyone. But theoretically except for the request of changing lecturer, all other options can be fulfilled simultaneously by providing mkv format video for download. However, a MOOC needs its lecture videos online, so technically there is still a long way to go.

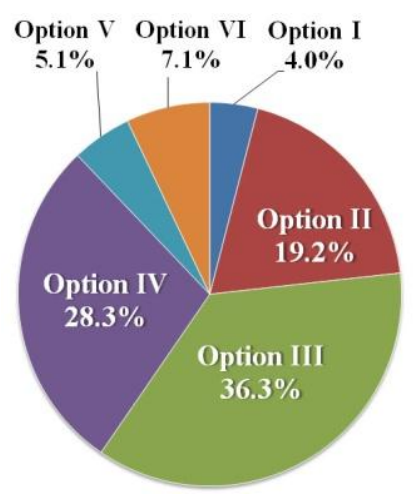

a) Full distribution

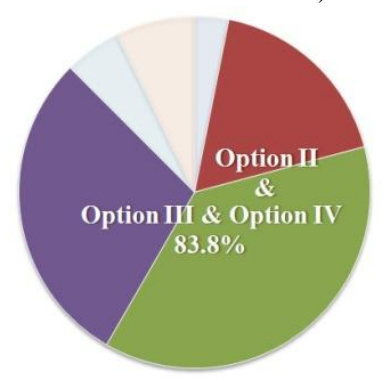

b) Who prefer subtitles

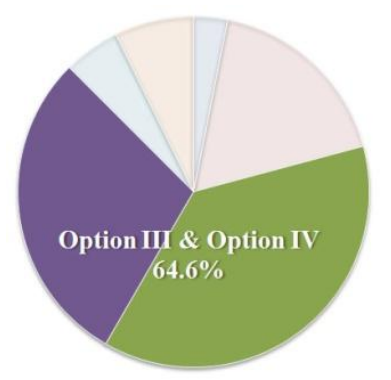

c) Who prefer bilingual subtitles Fig. 2. Users' preference on language delivery.

Since we cannot apply the multi-optional solution at once, bilingual subtitles is undoubtedly the best option we can offer at this time. We plan to implement this in the next course. However, the video is not the only thing that needs to be localized, the platform is also very important. With a well designed user interface in the mother language, the learning experience can be improved. Luckily, this task has been done on openHPI.cn.

\section{B. Adapting to the Cultural Difference}

Cultural difference is such a huge topic that it has been researched for centuries without a certain answer being found. But in the higher education area, Hofstede's model [15] has been widely applied to explain the culture-related phenomena within the classroom [16]. The discussion about this originally 4-dimensional - and then gradually extended to 6-dimensional - model has been fierce for decades, since the very beginning of its advent, including both critiques and acknowledgements [17]-[19].

One typical deduction from Hofstede's model is that in the teacher-student relationship, a teacher in the Confucian educational tradition plays a more dominant role in the teaching activities than a teacher in the Socratic educational tradition. China is the origin of Confucianism and deeply influenced by it [20], while western countries are considered to have inherited a great deal from Socrates [21], [22]. In our case, we offered the "Internetworking" course both on openHPI.de and openHPI.cn one after another, and with the course language of English and Chinese respectively. This gave us an excellent opportunity to observe the different behaviors from these two user groups. We address these groups as DEG-EN (openHPI.de group, English course) and CNG (openHPI.cn Group). 
TABLE IV: THE FORUM STATS FROM BOTH PLATFORMS

\begin{tabular}{|c|c|c|c|c|c|c|c|c|c|}
\hline & & & Total Topics & Total Posts & $\mathrm{P}$ by $\mathrm{S}$ & $\mathrm{P}$ by $\mathrm{T}$ & Respondents & S. R. & T. R \\
\hline \multirow{6}{*}{ openhpi.de } & \multirow{2}{*}{ Academic } & Sum & 165 & 435 & 374 & 61 & 375 & 322 & 53 \\
\hline & & Average & - & 2.64 & 2.27 & 0.37 & 2.27 & 1.95 & 0.32 \\
\hline & \multirow{2}{*}{ Non-Academic } & Sum & 118 & 214 & 164 & 50 & 187 & 140 & 46 \\
\hline & & Average & - & 1.81 & 1.39 & 0.42 & 1.58 & 1.19 & 0.39 \\
\hline & \multirow{2}{*}{ All } & Sum & 283 & 649 & 538 & 111 & 562 & 462 & 99 \\
\hline & & Average & - & 2.29 & 1.90 & 0.39 & 1.99 & 1.63 & 0.35 \\
\hline \multirow{6}{*}{ openhpi.cn } & \multirow{2}{*}{ Academic } & Sum & 51 & 114 & 58 & 56 & 101 & 52 & 49 \\
\hline & & Average & - & 2.24 & 1.14 & 1.10 & 1.98 & 1.02 & 0.96 \\
\hline & \multirow{2}{*}{ Non-Academic } & Sum & 51 & 98 & 53 & 45 & 84 & 40 & 44 \\
\hline & & Average & - & 1.92 & 1.04 & 0.88 & 1.65 & 0.78 & 0.86 \\
\hline & \multirow{2}{*}{ All } & Sum & 102 & 212 & 111 & 101 & 185 & 92 & 93 \\
\hline & & Average & - & 2.08 & 1.09 & 0.99 & 1.81 & 0.90 & 0.91 \\
\hline
\end{tabular}

Some researchers claim that it is inappropriate to equate culture with nationality, or to use terms like "the Chinese learner" to categorize the student group irrespective of age, gender or educational background [23], [24]. We also encountered this problem. Since simplified Chinese is the course language of openHPI.cn, the majority of $\mathrm{CNG}$ is guaranteed to be Chinese who live in mainland China. We also noticed that several course registrants accessed our course from Malaysia, Germany and the US. As for DEG-EN, the registrants come from all over the world, but with a higher proportion from Germany, which is quite understandable because openHPI.de is Germany-based. Based on all of these facts, we have limited our discussion to a general level when analyzing the user behavior from DEG-EN and CNG.

Observing the interaction between the learners and the teaching team is the key point in proving whether the deduction from Hofstede's model is suitable for our case. Such interactions within "Internetworking" primarily took place in the course-connected discussion forum, where all course participants and the teaching assistants could communicate with each other freely on both academic issues and non-academic problems. Table IV shows the statistics collected from the forums, including the numbers of total topics, responses, respondents per topic and their composition (student or teacher). Furthermore, the topics started by the teaching team, repeated posts and the responses solely expressing gratitude have been filtered.

From Table IV we can easily see that DEG-EN learners are more active in the forum than the $\mathrm{CNG}$ learners. On the average level, an academic issue would attract 1.95 students to post 2.27 responses while the relevant stats for a non-academic problem are 1.19 and 1.39. In contrast, we see that in CNG, either the number of student respondents or the number of student-posted responses is much smaller.

Since the average numbers of total responses on both platforms do not differ so much (2.29 in DEG-EN and 2.08 in $\mathrm{CNG})$, it is clear that the teaching team from openHPI.cn posted a lot. Fig. 3 clearly illustrates this fact. The teaching team on openHPI.de obviously posted less than the students in DEG-EN, around $20 \%$ to $80 \%$ proportionally. However, this proportion on openHPI.cn is generally even.

Another fact that can be extracted from Table IV is the function difference of the two teaching teams. The openHPI.de teaching team played the role of administrators a bit more than teachers by posting more on non-academic problems than academic issues. The openHPI.cn teaching team was just on the opposite.

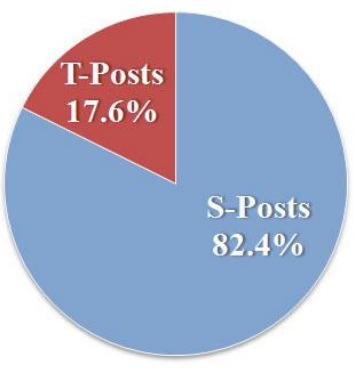

a) DEG-EN on openHPI.de

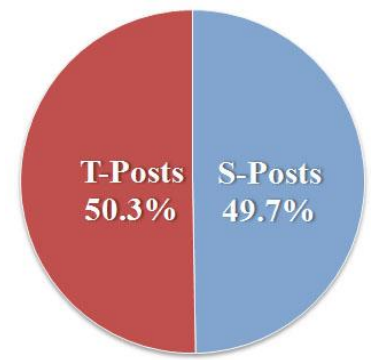

b) CNG on openHPI.cn
Fig. 3. The proportions of teacher and student posts in the forum.

Due to the different number on total enrollees in both "Internetworking" courses, the number of active users in CNG is also smaller than DEG-EN, which makes our comparison not completely convincing. But we can still conclude that the course participants from $\mathrm{CNG}$ are more reserved in public discussion than DEG-EN. This requires the teaching team to devote more efforts to keep the forum running. In other words, the openHPI.cn teaching team plays a more important role in the interaction with course participants than the openHPI.de teaching team. This fact suggests the correctness or rationality of the deduction deriving from Hofstede's model on our case.

TABLE V: THE QUESTIONNAIRE ITEM ON THE WILLINGNESS TO PARTICIPATE THE FORUM DISCUSSION

\begin{tabular}{lcc}
\hline \hline Do you want to join the discussion in our forum? & Count & Ratio \\
\hline (I) Absolutely yes. I want to be involved & 53 & $53.5 \%$ \\
(II)Yes. but only with the teaching assistants & 11 & $11.1 \%$ \\
(III)Yes. but only with fellow learners & 7 & $7.1 \%$ \\
(IV) I prefer to be a silent watcher in forum & 27 & $27.3 \%$ \\
(V) I don't use the forum function & 1 & $1.0 \%$ \\
\hline \hline
\end{tabular}

In addition, we designed an investigation item about the willingness of forum participation to take part in the feedback questionnaire. The collected result can be found in Table V. Less than $30 \%$ of the respondents clearly express that they didn't want to be involved into any kind of discussion. And another approximate $20 \%$ of the respondents chose a certain group of people when discussing, either the teaching 
assistants or fellow learners. Only a bit more than half of the respondents are totally open to the forum discussion. But being open doesn't mean the certainty of participation.

Unfortunately we didn't ask the same question regarding DEG-EN, so we can't make a comparison about it. But since the questionnaire respondents are already the most active user group of all course enrollees, the comparatively low participation rate in the forum discussion is not difficult to explain. Maybe it is just the typical Chinese personality to be modest and cautious that prevent the CNG, but that is something cannot or even should not be changed.

Based on all our findings in the MOOC "Internetworking", along with some other researchers' previous work, which compared the students' behaviors in both theoretical and practical ways in China and UK [19], [25], we would suggest that in educational activities, the influence of the culture difference does exist. As for MOOCs, what can we do to make our courses better when the majority of potential participants belong to a specific culture? At least for the Chinese or Chinese-speaking learners, a greater investment of time from the teaching team would help. We can consider this as an initial step of adapting to the culture difference which can be further involved in the MOOC localization effort.

\section{Other Observations}

In this chapter we would like present some other observations and analysis based on "Internetworking" courses. As shown in Table I, the earliest "Internetworking" course was the one offered in 2012 on openHPI.de with German as the teaching language. By that time the teaching team had also implemented a questionnaire, which was more focusing on platform related questions and is therefore quite different content-wise than our recently launched questionnaire on CNG. But there are still a few shared investigation items on the purpose of participation or learning habits. Thus we would like to make a comparison whereby we address the user group of the German-speaking "Internetworking" as DEG-DE (openHPI.de Group, Deustch Kurs).

Firstly we want to talk about the purpose of attending the course. As depicted in Fig. 4, the two user groups, DEG-DE and $\mathrm{CNG}$, generally have the similar reasons for participation. The interest in information technology is the overwhelming No. 1 reason for all the users to enroll in the course. This is followed by curiosity about the MOOC, a professional requirement and the pursuit of a certificate. One comparatively large difference appears in connection with the purpose of a professional requirement. As this is the answer given by $55 \%$ in DEG-DE but only $35 \%$ in $\mathrm{CNG}$, it leads us to a hypothesis, that there are more IT-professionals in DEG-DE than CNG.

The following facts seem to support this hypothesis. Fig. 5 shows that the DEG-DE tended to spend more time on the course than CNG. And more users in $\mathrm{CNG}$, as suggested in Fig. 6, preferred to finish all the weekly tasks in a single attempt. Both facts suggest that in comparison with $\mathrm{CNG}$, users in DEG-DE were more devoted to the course, by arranging the learning schedule more constantly and intensively. This might result from the higher proportion of IT professionals who have stronger motives, and also explains why the completion rate of DEG-DE is beyond $16 \%$, as shown in Table I, while in CNG this rate is only around $5 \%$.

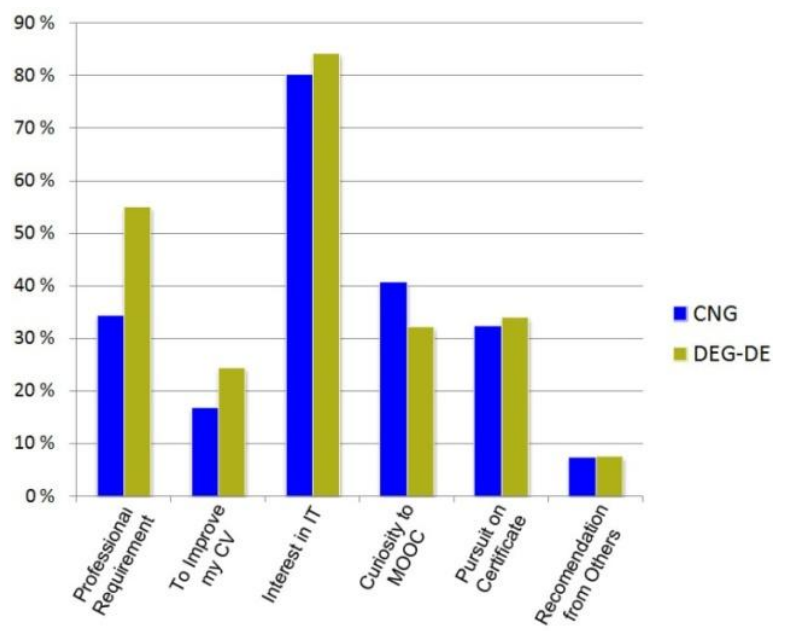

Fig. 4. The comparison of users' learning purpose.

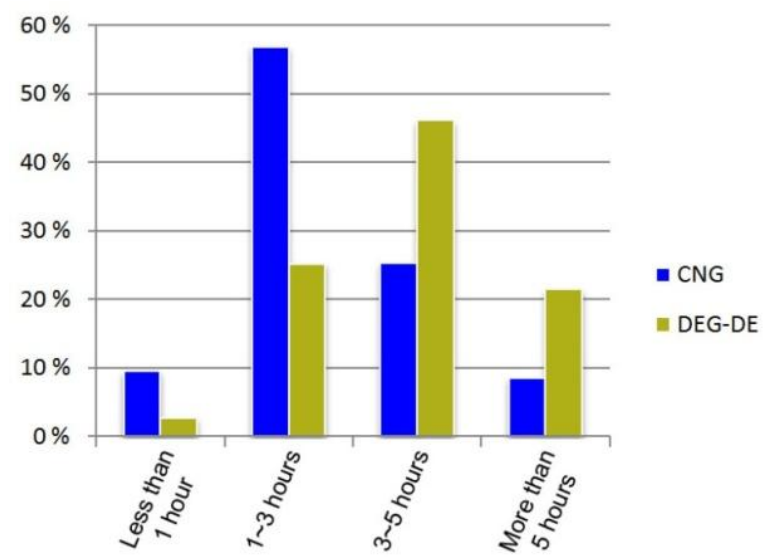

Fig. 5. The comparison of users' learning hours per week.

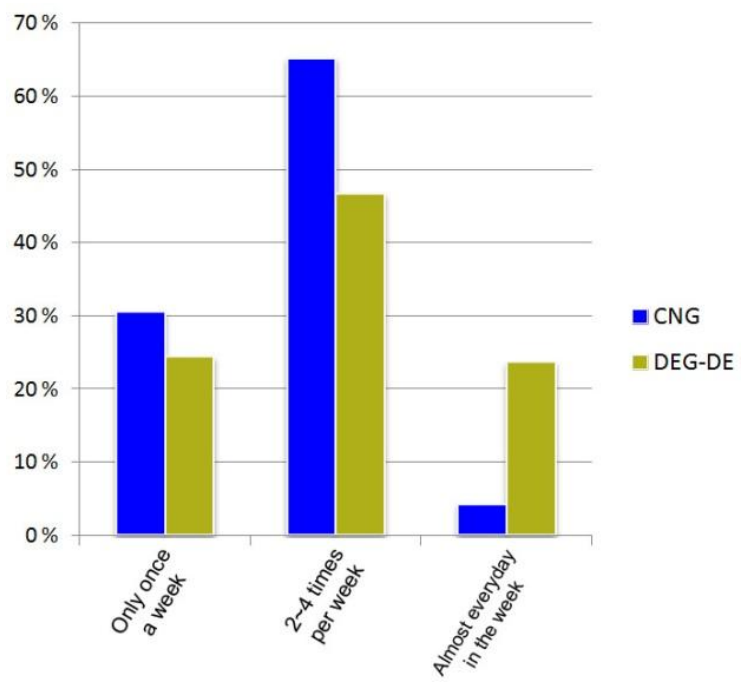

Fig. 6. The comparison of users' learning frequency.

In general, a comparatively low average completion rate of MOOC [10], reported from $6.5 \%$ to $10 \%$ by some researchers [26], [27], is one of the most bothering issues for the MOOC providers. When "Internetworking" offered on openHPI.de, no matter in German or English, an approximate completion rate of $16 \%$ was quite a success. But with the same course content, the rate dropped drastically on openHPI.cn, which means we still need to improve a lot. 
Finally we would like to present here a small sample of user's feedback. Based on their opinions, we are made aware of the advantage and disadvantage in the MOOC localization process, which will also guide the direction of our future efforts:

"I appreciate that there's one e-mail reminder per week to keep me in line and having Chinese-speaking teaching assistants to talk with", quote by questionnaire respondent No. 20, from Guangzhou, Guangdong, China.

"I like the high resolution lecture videos. When learning IT, my English is also improving", quote by questionnaire respondent No. 45, from Qinhuangdao, Hebei, China.

"The bilingual course helps me improve my Chinese, but I hope in next course there will be bilingual subtitle, sometime the translation in this course confuses me", quote by questionnaire respondent No. 49, from Kuala Lumpur, Malaysia.

"I hope openHPI can establish some kind of cooperation with Chinese universities. And MORE COURSES PLEASE", quote by questionnaire respondent No. 26, from Beijing, China.

\section{CONCLUSION AND FUTURE WORK}

In this paper we mainly shared our experience and efforts as a teaching team involved in the localized MOOC "Internetworking". After briefly introducing the development of MOOC and our MOOC platform, openHPI, we analyzed the ambivalence of Chinese-speaking MOOC users and offered our solution for localization. Then we talked about our attempt in detail, starting with the language issue and followed by the impact of culture difference as well as other observations.

The aim of MOOC is to spread knowledge to more people in this world with a better approach. Thus, one significant feature of MOOC is its internationalization. However, what we suggest in this paper is localization and we believe there is no contradictory between the two. In our eyes, the goal of MOOC localization is to help a specified user group Chinese-speaking learners in our case - to share the joy of obtaining the knowledge delivered by originally internationalized courses.

Some research works have mentioned the concept of "LOOC" [28]-[30]. But no matter how this ' $L$ ' is defined, whether "little", "locally" or even "localized", it doesn't represent our idea of MOOC localization. We have no intention of changing the ' $\mathrm{M}$ ' in MOOC, but we would like to make the ' $M$ ' more effective. In the future, more attempts will be applied to openHPI.cn, and we hope there will be more efforts toward localization, not only in connection with Chinese-speaking users, but also to facilitate people from all corners of this world.

\section{REFERENCES}

[1] A. McAuley, B. Stewart, G. Siemens, and D. Cormier, "Massive open online courses," Digital Ways of Knowing and Learning, 2010.

[2] L. Pappano, "The year of the MOOC," The New York Times, vol. 2, no. 12. p. 2012, 2012.

[3] C. Meinel, M. Totschnig, and C. Willems, "Openhpi: Evolution of a mooc platform from $1 \mathrm{~ms}$ to soa," in Proc. the 5th International Conference on Computer Supported Education, Germany, vol. 5, 2013.
[4] K. G. Rice, J. S. Ashby, and K. J. Preusser, "Perfectionism, relationships with parents, and self-esteem," Individual Psychology: Journal of Adlerian Theory, Research \& Practice, 1996.

[5] T. Liyanagunawardena, S. Williams, and A. Adams, "The impact and reach of MOOCs: a developing countries' perspective," Elearning Papers, no. 33, 2013.

[6] S. Boga and R. McGreal, "Introducing MOOCs to Africa," 2014.

[7] X. Gu, Y. Hu, and H. Cai, "Appeal and response to the development of moocs localization in china," Journal of Distance Education, vol. 5, p. $1,2013$.

[8] J. C.-C. Chen, "Opportunities and challenges of MOOCs: Perspectives from Asia," 2013

[9] A. Cross, M. Bayyapunedi, D. Ravindran, E. Cutrell, and W. Thies, "Vidwiki: Enabling the crowd to improve the legibility of online educational videos," in Proc. the 17th ACM Conference on Computer Supported Cooperative Work \& Social Computing, ACM, 2014, pp. 1167-1175.

[10] L. Breslow, D. E. Pritchard, J. DeBoer, G. S. Stump, A. D. Ho, and D. Seaton, "Studying learning in the worldwide classroom: Research into edxs first MOOC," Research \& Practice in Assessment, vol. 8, pp. $13-25,2013$

[11] J. DeBoer, G. Stump, D. Seaton, and L. Breslow, "Diversity in MOOC students backgrounds and behaviors in relationship to performance in 6.002 x," in Proc. the Sixth Learning International Networks Consortium Conference, 2013.

[12] K. Bolton, "English in Asia, Asian Englishes, and the issue of proficiency," English Today, vol. 24, no. 2, pp. 3-12. 2008.

[13] J. Gil, B. Adamson, and A. Feng, "The English language in mainland china: A sociolinguistic profile," English Language Education across Greater China, pp. 23-45, 2011.

[14] N. Ostashewski, M. Thorpe, and D. Gibson, "Addressing the challenges of a bilingually delivered online course: design and development of the Australia china trade (act) MOOC," in Proc. World Conference on e-Learning in Corporate. Government. Healthcare and Higher Education, vol. 2013, no. 1, 2013, pp. 1284-1289.

[15] G. Hofstede, "Dimensionalizing cultures: The hofstede model in context," Online Readings in Psychology and Culture, vol. 2, no. 1, p. $8,2011$.

[16] G. Hofstede, "Cultural differences in teaching and learning," International Journal of intercultural relations, vol. 10, no. 3, pp. 301-320, 1986.

[17] M. Søndergaard, "Research note: Hofstede's consequences: a study of reviews, citations and replications," Organization studies, vol. 15, no. 3, pp. 447-456, 1994.

[18] B. McSweeney, "Hofstedes model of national cultural differences and their consequences: A triumph of faith-a failure of analysis," Human relations, vol. 55, no. 1, pp. 89-118, 2002.

[19] P. Signorini, R. Wiesemes, and R. Murphy, "Developing alternative frameworks for exploring intercultural learning: a critique of hofstede's cultural difference model," Teaching in Higher Education, vol. 14, no. 3, pp. 253-264, 2009.

[20] X. Yao, An Introduction to Confucianism, Cambridge University Press, 2000.

[21] A. G. Rud, "The use and abuse of socrates in present day teaching," Education Policy Analysis Archives, vol. 5, no. 5, pp. 1068-2341, 1997.

[22] A. Mintz, "From grade school to law school: Socrates legacy in education," A Companion to Socrates, p. 476, 2006.

[23] P. Kennedy, "Learning cultures and learning styles: Myth understandings about adult (Hong Kong) Chinese learners," International Journal of Lifelong Education, vol. 21, no. 5, pp. 430-445, 2002.

[24] T. Grimshaw, "Problematizing the construct of the Chinese learner: Insights from ethnographic research," Educational Studies, vol. 33, no. 3, pp. 299-311, 2007.

[25] Q. Gu and M. Schweisfurth, "Who adapts? Beyond cultural models of thechinese learner," Language, Culture and Curriculum, vol. 19, no. 1, pp. 74-89, 2006.

[26] I. Nawrot and A. Doucet, "Building engagement for MOOC students: introducing support for time management on online learning platforms," in Proc. the companion publication of the 23rd international conference on World Wide Web Companion, 2014, pp. 1077-1082.

[27] K. Jordan, "Initial trends in enrolment and completion of massive open online courses," The International Review of Research in Open and Distance Learning, vol. 15, no. 1, 2014.

[28] A. Chauhan, "Massive open online courses (MOOCs): Emerging trends in assessment and accreditation," Digital Education Review, no. 25 , pp. 7-17, 2014.

[29] J. K. Parker, "Opportunities and challenges of a locally open online course (LOOC)," 2013. 
[30] G. Drexhage, "Ubc library: Fall update," BCLA Browser: Linking the Library Landscape, vol. 5, no. 4, 2013.

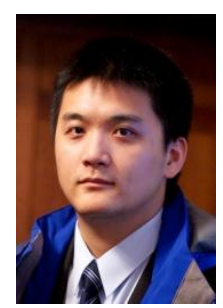

Xiaoyin Che was born on January 2, 1987 in Beijing, China. He entered university in 2005 and received his bachelor degree from College of Computer Science, Beijing University of Technology (BJUT) in 2009, majored in computer science and technology. Then he started his master program in multimedia and intelligent software technology laboratory, College of Computer Science, BJUT and received the degree in 2012. He is currently a $\mathrm{PhD}$ student in the chair of internet technologies and systems, Hasso Plattner Institute, Potsdam, Germany.

He previously researched in video coding standards, image processing and compressive sensing. Now he's working with video analysis, document processing, e-learning and $\mathrm{MOOC}$.

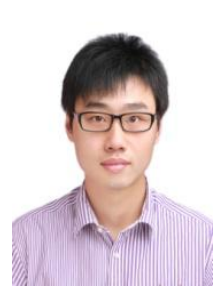

Sheng Luo is now working on his doctoral research in Hasso Plattner Institute, Potsdam University. He received a bachelor of engineering in computer science and technology from Shanghai University in 2011, a master of engineering from Waseda University in 2013 and a master of engineering in computer science and technology from Shanghai University in 2014. His research interests include e-learning, embedded system, wireless sensor network etc.

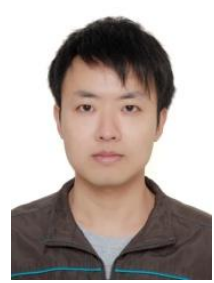

Cheng Wang was born in 1987. He obtained his bachelor of management degree from Shandong Jianzhu University and master of science degree from Sichuan University in 2010 and 2013 respectively. He is currently working toward $\mathrm{PhD}$ degree in Hasso Plattner Institute, University of Potsdam, Germany. His research interests include machine learning, deep learning, computer vision, multimedia retrieval and MOOC

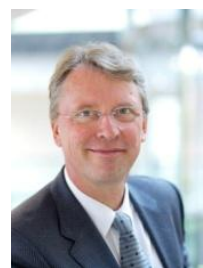

Sc. nat.

$\mathrm{He}$ is the scientific director and CEO of the Hasso Plattner Institute for Software Systems Engineering GmbH (HPI), Potsdam, Germany, since 2004. Previously he worked in University of Saarbrücken and University of Paderborn, and became a full professor (C4) for computer science at the University of Trier. His research interests are focused on Internet and information security, web 3.0, semantic web, social and service web and the domains of e-learning, tele-teaching and tele-medicine. Christoph Meinel is author/co-author of 9 books and 4 anthologies, as well as editor of various conference proceedings. More than 400 of his papers have been published in high-profile scientific journals and at international conferences. 